\title{
Impact of a diabetes remote monitoring program on medication adherence
}

\author{
Kiraat D Munshi, PhD; Kyle Amelung, PharmD, BCPS; Callie S Carter, PharmD; Roberta James, MStat; \\ Bimal R Shah, MD, MBA; and Rochelle R Henderson, PhD
}

\section{What is already known about this subject}

- Diabetes remote monitoring (DRM) programs have proliferated and been implemented at scale as mobile health technologies have evolved over the past decade.

- DRMs have reported glycemic improvements; however, the effect of remote monitoring and mobile health technologies on adherence has been mixed.

- Most studies have been conducted among small samples of patients using different technologies and often with adherence estimated using selfreported questionnaires.

\section{What this study adds}

- DRM participation and engagement were associated with higher medication adherence among people with diabetes compared with those not participating in a DRM program.

- Longer engagement in a DRM program were associated with greater improvements in medication adherence.

- Study findings have important implications for payers and patients related to improved health outcomes through digital health solutions.

\section{Author affiliations}

Kiraat D Munshi, PhD, Health Services Research; Kyle Amelung, PharmD, BCPS, Clinical Solutions; Callie S Carter, PharmD, Office of Clinical Evaluation \& Policy; and Rochelle R Henderson, PhD, Health Services Research, Express Scripts, Memphis, TN. Roberta James, MStat, Clinical Analytics, Livongo, Mountain View, CA. Bimal R Shah, MD, MBA, Chief Medical Officer, Livongo, Mountain View, CA, and Duke University School of Medicine, Durham, NC.

\section{AUTHOR CORRESPONDENCE:}

Kiraat D Munshi, 901.381.7277;

kdmunshi@express-scripts.com

J Manag Care Spec Pharm 2021;27(6):724-31

Copyright (C2021, Academy of Managed Care Pharmacy. All rights reserved.

adherence measured as proportion of days covered (PDC) in the 365 days following first BG check was examined as a continuous and binary outcome measure (PDC $>80 \%$ or adherent vs $<80 \%$ or nonadherent). Multivariable linear and logistic regression were conducted to examine differential magnitude in adherence and likelihood of being adherent, respectively.

RESULTS: The final sample consisted of 6,002 exposure and 12,004 nonexposure group patients. DRM participants who were ever engaged had a $4.5 \%$ higher adherence rate $(P<0.001)$ and $42 \%$ higher odds of being adherent $(P<0.001)$ in the period after engagement compared with non-DRM participants. Sensitivity analyses showed that patients engaged continuously (>1 BG check 
per week) for 3, 6, and 12 months had $5.1 \%, 5.2 \%$, and $6.4 \%$ higher adherence rates, respectively $(P<0.001)$, and $52 \%, 64 \%$, and $98 \%$ higher odds of being adherent, respectively $(P<0.001)$, compared with non-DRM participants.

CONCLUSIONS: The study findings offer evidence that DRM engagement is associated with higher odds of medication adherence. DRM solutions that provide access to glucose test results, personalized coaching, educational resources, and lower testing supply cost can also influence adherence. Our findings have important implications for payers and patients related to improved health outcomes due to higher medication adherence.

Over 120 million US adults are living with diabetes or prediabetes, with diabetes being the seventh leading cause of death in the United States in 2017., ${ }^{1,2}$ As a chronic condition highly dependent on self-management, nearly half of all individuals with diabetes are reported to have poor glycemic control. ${ }^{3}$ Optimal management of type 2 diabetes mellitus (T2DM) involves a variety of therapies that involve changes in lifestyle, including diet and physical activity, as well as pharmacotherapy, to improve metabolic control in most individuals., ${ }^{4,5}$

Treating T2DM typically often requires multiple medications, and medication adherence has been associated with improved risk factor management, lower hospitalization rates, lower total health care costs, and improved longevity. ${ }^{6-11}$ However, many individuals with T2DM lack tools for and knowledge about self-management of their diabetes and medications, resulting in suboptimal medication management. Diabetes remote monitoring (DRM) programs have proliferated and been implemented at scale as mobile health technologies have evolved over the past decade to address these gaps in self-management for people with diabetes. ${ }^{12}$ DRM programs have reported glycemic improvements; however, outcomes vary by program, age, and sex. ${ }^{13-16}$ Evidence is increasing regarding the effect of DRM programs on medication adherence ${ }^{17}$; however, the effect of remote monitoring and mobile health technologies on adherence has been mixed, with some trials showing improvements in adherence, while others have not found statistically significant improvements. ${ }^{18,19}$ Most studies have been conducted among small samples of patients using different technologies and often with adherence estimated using self-reported questionnaires.

We are unaware of previously published studies in a large population of people with T2DM to examine the influence of these technologies on oral antidiabetic drug (OAD) medication adherence using pharmacy claims data. As a result, we sought to examine the association between participation in a DRM digital health service-which incorporated a cellular connected glucometer, free strips and supplies, a smartphone application, and access to virtual sessions with certified diabetes educators (CDEs)-and medication adherence.

\section{Methods}

\section{STUDY DESIGN AND SAMPLE}

This study was a retrospective, propensity score-matched cohort study that used deidentified member enrollment and pharmacy administrative claims from a large national pharmacy benefit manager for 2014-2019. Inclusion was limited to commercially insured adults and retirees aged 18 years or older as of their index dates during which they were included in the study. Members enrolled in the DRM program who submitted at least 1 blood glucose (BG) check between April 13, 2015, and March 31, 2018, were included in the study. The date of the first BG check reported was defined as the index date, and the index period was defined as a 90-day period from the index date.

Members were included if they filled 2 or more 30-day adjusted OAD prescription claims in the index period (herein referred to as people with diabetes or participants). The diabetes therapy classes included in this study were metformin, sulfonylureas, thiazolidinediones, meglitinides, alpha-glucosidase inhibitors, dipeptidyl peptidase-4 inhibitors, and sodium glucose co-transporter inhibitors. Participants who were not continuously enrolled in their health plans for a period of 180 days before and 365 days after the index date, and those who filled only insulin or noninsulin injectable drug claims were excluded from the study. The 180-day pre-index period was referred to as the lookback index period, and the 365-day period was the measurement period (Figure 1).

Members enrolled in commercial insurance plans contracted to receive DRM services who had a confirmed diagnosis of T2DM were offered the opportunity to opt into the program. All participants in the DRM program received a 2-way cellular connected glucometer with unlimited strips and testing supplies, a smartphone application (iOS and Android) that provided information on the results of BG checks, and health literacy information related to T2DM. This diabetes-related content was accredited by the American Diabetes Association and the Association of Diabetes Care and Education Specialists (ADCES), and participants had access to unlimited virtual live coaching by CDEs who had clinical backgrounds as nutritionists, dietitians, or exercise therapist/physiologists. Specific program 


\section{FIGURE 1 Study Timeline}

Study Period: Members must be continuously enrolled

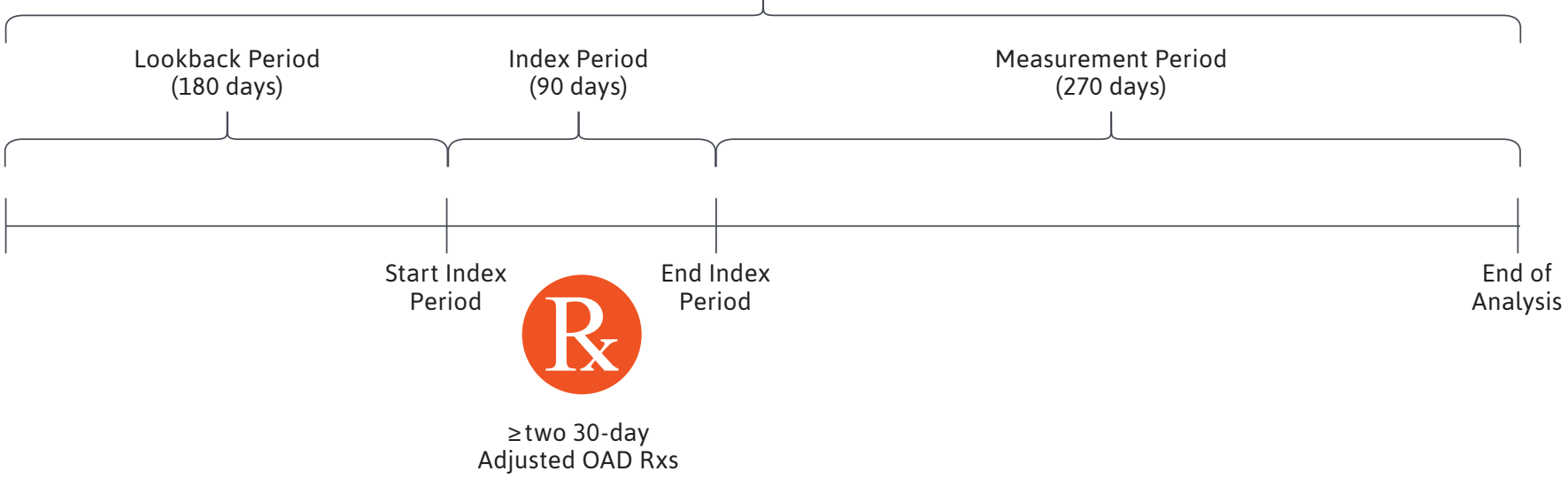

$O A D=$ oral antidiabetic drug; $R x=$ prescription claim .

aspects built on the ADCES 7 Self Care behaviors practice guidelines to promote medication adherence were included and accredited by ADCES in the DRM program. ${ }^{8}$

The comparison group included commercially insured adults and retirees who were enrolled in a health plan that did not implement any DRM program through the pharmacy benefit manager. An 8:1 propensity score matching was conducted to match the DRM enrollees with the comparison group members on demographic variables (age, sex, and geographic region). The matched comparison group members were then assigned the index date of the DRM enrollees they matched to, and further inclusion and exclusion criteria were applied. The final sample of comparison group members with 2 or more 30-day adjusted OAD prescription claims in the index period and continuous eligibility were then matched once again to the DRM enrollees on all covariates listed in the next section using a 2:1 propensity score-matching approach.

\section{STUDY VARIABLES}

Medication adherence, measured as proportion of days covered (PDC), was the outcome of interest. PDC measures the proportion of the study duration during which patients had their OADs on hand. PDC was calculated as both continuous and binary measures, where a PDC of less than $80 \%$ was considered suboptimal (reference variable), and patients with PDC of $80 \%$ or more were considered adherent.

The covariates examined in this study were age (in years) as of index date, sex (women [reference variable], men), region, previous medication adherence, disease burden proxy score, total pharmacy out-of-pocket costs, number of OAD classes filled during the index period, and number of insulin drugs filled during the study period. The region variable was based on the geographic census regions of Northeast (reference variable), Midwest, South, and West.

Previous medication adherence was the PDC for each patient in the study calculated over the 180-day lookback period. This variable was included in our analyses to account for previous adherence behavior effect, since past behavior is a good marker for predicting future behavior. ${ }^{20}$

Patient overall disease burden was a proxy calculated from prescription claims data and was defined as the number of unique 2-digit Generic Product Identifiers indicating drug therapy classes used by the patient. Number of index OAD classes and number of insulin medications were included to account for differential severity of diabetes between the 2 study groups.

\section{ANALYSIS}

Bivariate comparison of outcomes in the propensity scorematched study groups was conducted using the t-test (continuous variables) and McNemar's test (categorical variables). Differences in continuous PDC were analyzed using multivariable linear regression, while multivariable logistic regression was used for the binary PDC measure to estimate the odds of being adherent. SAS version 9.4 was used for data processing and analyses (SAS Institute). 


\section{FIGURE 2 Sample Selection}

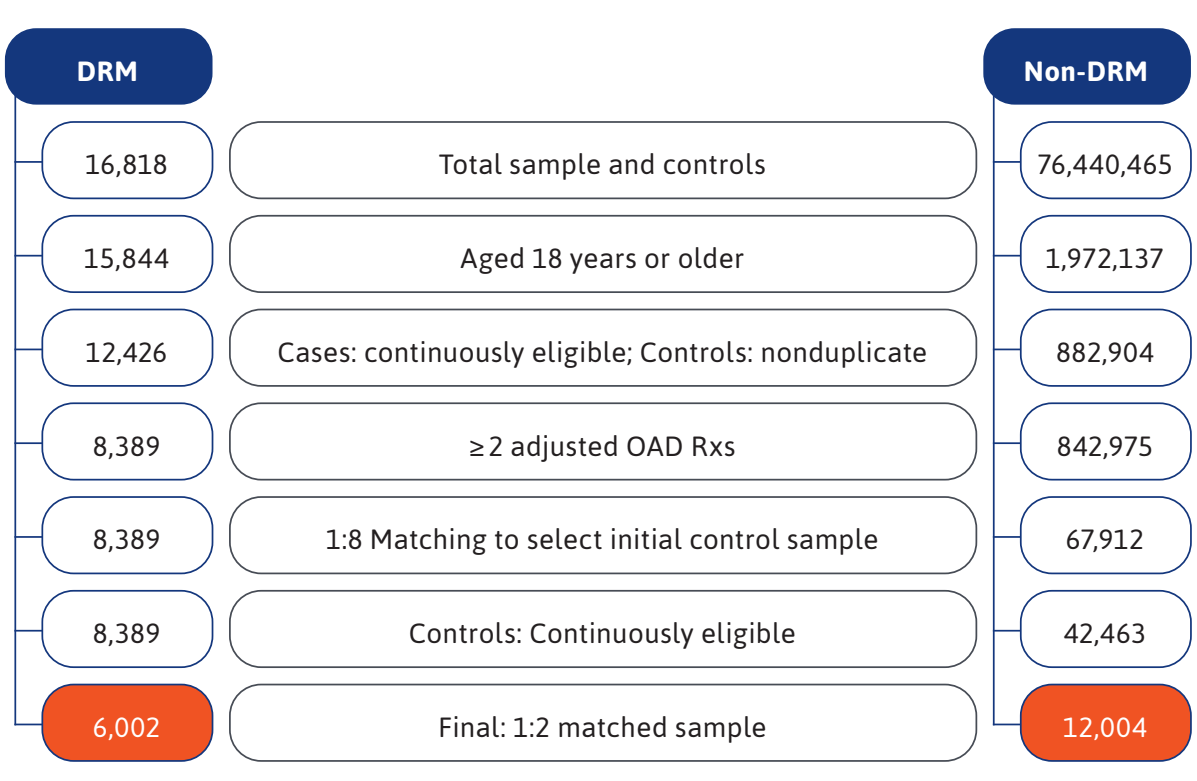

$D R M=$ diabetes remote monitoring; $O A D=$ oral antidiabetic drug; $R x=$ prescription.

\section{SENSITIVITY ANALYSIS}

We conducted 3 sets of analyses among patients with varying levels of engagement with the DRM program. Adherence was examined among DRM patients who were continuously engaged with the DRM program for at least 3 months, at least 6 months, and at least 12 months. Continuous engagement was defined as reporting at least $1 \mathrm{BG}$ reading per week during all weeks in the month. The hypothesis was that longer engagement with the DRM program would be associated with stronger associations with increase in medication adherence.

\section{Results}

A total of 16,818 DRM enrollees and over 76 million members in the comparison group were examined for inclusion in this analysis. The final 2:1 propensity score-matched sample included 6,002 DRM participants who reported at least $1 \mathrm{BG}$ check during the study period and 12,004 non-DRM participants (Figure 2). The demographic and health characteristics of the study groups are displayed in Table 1. After propensity score matching, the 2 study groups did not have any statistically significant differences for these characteristics. Average age of the study sample was over 54 years, and more than $57 \%$ of patients in each group were women.

Participants were mostly prevalent users of OADs $(\geq 1$ OAD claim in lookback period), and about $40 \%$ of patients in both groups were from the South. Average disease burden proxy score was over 6.6 and previous adherence of $60.7 \%$, while both groups of participants had more than $\$ 770$ on average in total out-of-pocket pharmacy costs in the lookback period. They filled 1.59 OAD classes during the index period, and about 1 insulin drug during the study period.
Table 2 displays the bivariate analysis results. Average adherence in the measurement period was higher by nearly $5 \%$ for DRM participants compared with non-DRM participants (85.11\% vs $80.69 \%, \mathrm{P}<0.001)$. A difference of $6.1 \%$ between DRM and non-DRM groups was observed when examining the proportion of participants who were adherent ( $\mathrm{PDC} \geq 80 \%$ ). While $73.8 \%$ of people with diabetes were adherent in the DRM group, only $67.7 \%$ were adherent in the non-DRM group $(\mathrm{P}<0.001)$.

Multivariable regression analyses results are displayed in Table 3 . After adjusting for all demographic and health characteristics, average adherence was higher by $4.5 \%$ among the DRM group compared with the non-DRM group $(P<0.001)$. When examining the likelihood of being adherent, the DRM group was found to have $42 \%$ higher odds of being adherent to OADs after participating in the DRM program compared with the matched non-DRM counterparts over the same time period (odds ratio $=1.42$, 95\% CI=1.32-1.53; $\mathrm{P}<0.001$ ).

Our sensitivity analyses indicated that participants engaged for longer periods in the DRM program had higher improvements in adherence (Supplementary Figure 1, available in online article). We examined adherence among a subset of DRM participants who were enrolled continuously for at least 3 months $(\mathrm{n}=4,246)$, at least 6 months $(\mathrm{n}=2,632)$, and at least 12 months $(\mathrm{n}=1,019)$. The adherence was compared with their 2:1 propensity score-matched nonDRM group participants. Participants engaged continuously for at least 3 , at least 6 , and at least 12 months in the DRM program had $5.0 \%, 5.2 \%$, and $6.4 \%$ higher adherence, respectively, compared with matched non-DRM participants. Similarly, the odds of being adherent were $52 \%, 64 \%$, and 


\begin{tabular}{|c|c|c|c|c|c|}
\hline \multirow[b]{2}{*}{ Characteristics } & \multicolumn{2}{|c|}{$\begin{array}{l}\text { DRM group } \\
(n=6,002)\end{array}$} & \multicolumn{2}{|c|}{$\begin{array}{l}\text { Non-DRM group } \\
(n=12,004)\end{array}$} & \multirow[b]{2}{*}{ P value $^{a}$} \\
\hline & n or mean & $\%$ or SD & $\mathrm{n}$ or mean & $\%$ or SD & \\
\hline Age (in years) & 54.17 & 8.83 & 54.43 & 9.10 & 0.070 \\
\hline \multicolumn{6}{|l|}{ Sex } \\
\hline Women & 3,450 & 57.48 & 6,932 & 57.75 & 0.733 \\
\hline Men & 2,552 & 42.52 & 5,072 & 42.25 & \\
\hline \multicolumn{6}{|l|}{ New user } \\
\hline No & 5,634 & 93.87 & 11,265 & 93.84 & 0.948 \\
\hline Yes & 368 & 6.13 & 739 & 6.16 & \\
\hline Disease burden proxy & 6.65 & 4.12 & 6.75 & 4.15 & 0.128 \\
\hline \multicolumn{6}{|l|}{ Region } \\
\hline Northeast & 987 & 16.44 & 2,052 & 17.09 & 0.541 \\
\hline Midwest & 1,970 & 32.82 & 3,853 & 32.1 & \\
\hline South & 2,408 & 40.12 & 4,784 & 39.85 & \\
\hline West & 637 & 10.61 & 1,315 & 10.95 & \\
\hline Patient OOP cost & $\$ 793.00$ & $\$ 959.20$ & $\$ 771.50$ & $\$ 1,067.70$ & 0.187 \\
\hline Previous adherence & 60.66 & 29.35 & 60.90 & 29.28 & 0.600 \\
\hline Index drug classes, n & 1.59 & 0.74 & 1.59 & 0.76 & 0.733 \\
\hline Insulins, n & 1.21 & 2.82 & 1.20 & 3.32 & 0.783 \\
\hline \multicolumn{6}{|c|}{$\begin{array}{l}{ }^{a} A P \text { value of }<0.05 \text { indicated that differences between DRM and non-DRM participants were statistically } \\
\text { significant. } \\
\text { DRM = diabetes remote monitoring: } O O P=\text { out of pocket. }\end{array}$} \\
\hline
\end{tabular}

\section{TABLE 2 Bivariate Analysis of Unadjusted Medication Adherence Outcomes}

\begin{tabular}{|c|c|c|c|c|c|c|}
\hline \multirow[b]{2}{*}{ Characteristics } & \multirow[b]{2}{*}{ Groups } & \multicolumn{2}{|c|}{$\begin{array}{l}\text { DRM group } \\
(n=6,002)\end{array}$} & \multicolumn{2}{|c|}{$\begin{array}{l}\text { Non-DRM group } \\
(n=12,004)\end{array}$} & \multirow[b]{2}{*}{ P value } \\
\hline & & n or mean & $\%$ or SD & n or mean & $\%$ or SD & \\
\hline PDC, \% & & 85.11 & 20.66 & 80.69 & 24.27 & $<0.001$ \\
\hline \multirow{2}{*}{ Adherent $t^{b}$} & No & 1,575 & 26.24 & 3,883 & 32.35 & \multirow{2}{*}{$<0.001$} \\
\hline & Yes & 4,427 & 73.76 & 8,121 & 67.65 & \\
\hline
\end{tabular}

ap value of $<0.05$ indicated that differences between DRM and non-DRM groups were statistically significant.

bPeople with diabetes were considered adherent if they had an average PDC of $80 \%$ or higher. $D R M=$ diabetes remote monitoring; $P D C=$ proportion of days covered.

almost twice higher for the DRM group engaged continuously for at least 3 , at least 6 , and at least 12 months, respectively, compared with the matched non-DRM counterparts. All of these differences were statistically significant between both groups $(\mathrm{P}<0.001)$.

\section{Discussion}

To our knowledge, our findings represent the largest, real-world study of the effect that an integrated DRM has on improving medication adherence at 12 months. We found that, compared with a control group of matched individuals, those who enrolled in DRM had $4.5 \%$ higher adherence, with $42 \%$ higher odds of adherence to OADs in a period of 1 year after engagement. Longer engagement in the program led to higher adherence rates, suggesting a time dependent positive response to the DRM program as it relates to medication use. These findings suggest that a digital solution that offers a cellular connected glucometer, free strips and testing supplies, access to diabetes content through a smartphone application, and access to CDEs may also improve patient medication-taking behavior, beyond the direct effect on BG.

The World Health Organization notes that increasing adherence may have a greater effect on health than improvements in specific medical therapy. ${ }^{9}$ With about $37 \%$ of commercially insured people with diabetes being nonadherent to their medications, nonadherence is a serious challenge to chronic disease management and a driver of significant costs and comorbidity. ${ }^{10,21}$

The underuse of evidence-based therapies for chronic conditions imposes a substantial clinical and economic burden on patients and health care systems. ${ }^{22-24}$ For people with T2DM, this lack of adherence has dramatic effects on health and a substantial increase in mortality. ${ }^{25-28} \mathrm{Jha}$ et al found that patients with T2DM with improved medication adherence had a $13 \%$ reduction in the risk of hospitalization or emergency department (ED) visits, while a $15 \%$ increase in hospitalization and ED visits was associated with worsening adherence 


\section{TABLE 3 Multivariable Analysis Examining Average Adherence and Odds of Being Adherent Among DRM and Non-DRM Groups ${ }^{\text {a }}$}

\begin{tabular}{|c|c|c|c|c|c|c|c|c|}
\hline \multirow{3}{*}{$\begin{array}{c}\text { Groups } \\
\text { Non-DRM }\end{array}$} & \multicolumn{4}{|c|}{ Absolute difference in PDC (\%) } & \multicolumn{4}{|c|}{ Adherent (PDC $\geq 80 \%$ ) } \\
\hline & \multirow{2}{*}{$\begin{array}{c}\text { Beta estimate } \\
-\end{array}$} & \multicolumn{2}{|c|}{$95 \% \mathrm{Cl}$} & \multirow{2}{*}{$\begin{array}{c}\text { P value }^{\mathbf{b}} \\
-\end{array}$} & \multirow{2}{*}{$\begin{array}{c}\text { Odds ratio } \\
-\end{array}$} & \multicolumn{2}{|c|}{$95 \% \mathrm{Cl}$} & \multirow{2}{*}{$\frac{\text { P value }^{\text {b }}}{-}$} \\
\hline & & - & - & & & - & - & \\
\hline DRM & 4.54 & 3.88 & 5.19 & $<0.001$ & 1.42 & 1.32 & 1.53 & $<0.001$ \\
\hline
\end{tabular}

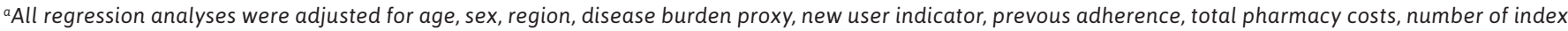
diabetes drug classes and number of concomitant insulins.

${ }^{b} P$ value of $<0.05$ indicated that differences between DRM and non-DRM groups were statistically significant.

$D R M=$ diabetes remote monitoring; $P D C=$ proportion of days covered.

over time. ${ }^{29}$ A systematic review of the economic impact of medication adherence and/or persistence on the overall cost of T2DM care found that the average total annual cost per patient ranged from $\$ 4,570$ to $\$ 17,338$, and medication adherence was inversely associated with total health care and hospitalization costs. ${ }^{30}$

Our study leveraged a multimodal approach to address the multiple barriers that exist. Before enrollment in the DRM program, there was little difference in the PDC rates between non-DRM and DRM participants, and both groups had similar adherence rates at $61 \%$, which is similar to previous estimates. ${ }^{17}$ Clients of enrollees in both arms had access to similar utilization management programs and clinical solutions. The intervention group received full and unlimited access to the DRM program. At the end of 12 months, there was a clinically meaningful and statistically significant additional increase in medication adherence in those individuals engaged in the DRM program.

The multimodal aspect of the DRM program to assist individuals with medication rituals and reminders probably contributed to the observed improvement in adherence relative to the non-DRM cohort. In addition, providing free strips as part of the DRM program (where after 90 strips, most coverage plans require individuals to pay out of pocket for additional strip refills within 90 days) could have served to offset financial barriers to paying for oral diabetes medications. ${ }^{31}$

The longitudinal nature and need for continuous monitoring in chronic disease management with concomitant reductions in the costs of technology and sensors has accelerated developments in telehealth and remote monitoring. Many of these innovations are starting to demonstrate the ability to improve clinical outcomes and reduce costs; however, the effect on medication adherence has been mixed. ${ }^{18,32,33}$ Studies have shown the positive effect of virtual health tools such as text messaging and telephonic support for medication adherence in diabetes, but there have not been high quality studies with suitable concurrent controls to examine how other technologies improve medication adherence. ${ }^{18}$ This limited observed efficacy may reflect the fact that many interventions do not adequately address each individual's unique barriers to adherence and/or only do so at a single point in time. ${ }^{34}$

In addition, among those interventions demonstrating success, many have not been widely adopted because of the substantial human resources required to sustain them. ${ }^{35}$ Choudhry et al conducted a cluster randomized trial of a multicomponent intervention to improve medication adherence in individuals with hypertension, diabetes, and hyperlipidemia. ${ }^{17}$ Their program randomized patients to a multicomponent intervention using telephone-delivered behavioral interviewing by trained clinical pharmacists, text messaging, pillboxes, and mailed progress reports and found that the intervention group improved PDC by $4.7 \%$ at 12 months compared with usual care. ${ }^{17}$ Our results in the DRM group compared with the control group resulted in similar findings despite differences in the approach, suggesting that multicomponent interventions that address different barriers are required to provide meaningful improvements in medication adherence rates in people with diabetes.

\section{LIMITATIONS}

Our study has some limitations. The retrospective study design examined associations and not established causality. Also, given the opt-in nature of the DRM program, more motivated and healthier individuals could have introduced selection bias into the DRM group. However, through propensity matching, balancing the analysis of the endpoints of interest and the observation that both groups had similar trajectories of improvement in PDC at 12 months, we reduced the influence of this bias in our reported results.

We did not include injectables or 
insulin in our analysis of medication adherence given the difficulty using claims data to monitor adherence and the dispensing nature and variable administration that is often required in patients self-managing their diabetes medications. Moreover, claims-based adherence estimation is not able to verify that patients have consumed their medications as prescribed. However, this method is widely used and accepted as a measure for adherence in health services research.

Our findings are important and provide timely evidence on the effect of a DRM program, given the proliferation of these solutions and the wider adoption and implementation of digital health technologies by payers and providers. They also highlight the need for ensuring longer engagement on an ongoing basis for achieving greater improvements in outcomes such as medication adherence.

\section{Conclusions}

Our study shows that engagement in a scalable, multicomponent DRM program is associated with clinically meaningful improvements in medication adherence in T2DM using oral diabetes medications. Future studies should examine the relationship of the DRM program's medication adherence improvement to clinical outcomes and cost reductions.

\section{DISCLOSURES}

Funding for this study was provided by Express Scripts. Munshi, Amelung, Carter, and Henderson are employed by Express Scripts. James and Shah are employed by Livongo, which provided the DRM solution.

\section{ACKNOWLEDGMENTS}

The authors thank Caroline A. Swift, PhD, and Haresh Bhatia, $\mathrm{PhD}$, for contributions to this study.

\section{REFERENCES}

1. Centers for Disease Control and

Prevention. National diabetes statistics report, 2020. Accessed May 15, 2020. https://www.cdc.gov/diabetes/pdfs/ data/statistics/national-diabetes-statistics-report.pdf

2. Murphy SL, Xu J, Kochanek KD, Arias E. Mortality in the United States, 2017. NCHS Data Brief. 2018;(328):1-8.

3. Ryan JG, Fedders M, Jennings T, Vittoria I, Yanes M. Clinical outcomes and incremental costs from a medication adherence pilot intervention targeting low-income patients with diabetes at risk of cost-related medication nonadherence. Clin Ther. 2014;36(12):1991-2002. doi: 10.1016/j.clinthera.2014.09.001

4. American Diabetes Association. 5. Facilitating behavior change and wellbeing to improve health outcomes: standards of medical care in diabetes2020. Diabetes Care. 2020;43(Suppl 1): S48-S65.

5. American Diabetes Association. 9. Pharmacologic approaches to glycemic treatment: standards of medical care in diabetes-2020. Diabetes Care. 2020;43(Suppl 1):S98-S110.

6. DiBonaventura M, Wintfeld N, Huang J, Goren A. The association between nonadherence and glycated hemoglobin among type 2 diabetes patients using basal insulin analogs. Patient Prefer Adherence. 2014;8:873-82.

7. Kirkman MS, Rowan-Martin MT, Levin R et al. Determinants of adherence to diabetes medications: findings from a large pharmacy claims database. Diabetes Care. 2015;38:604-09.

8. American Association of Diabetes Educators. An effective model of diabetes care and education: revising the AADE7 Self-Care Behaviors. Diabetes Educ. 2020;46(2):139-60.

9. Sabaté E. Adherence to long-term therapies: evidence for action. World Health Organization; 2003.

10. Burkhart PV, Sabate E. Adherence to long-term therapies: evidence for action. J Nurs Scholarsh. 2003;35:207.
11. Krass I, Schieback P, Dhippayom T. Adherence to diabetes medication: a systematic review. Diabet Med. 2015;32(6):725-37. doi: 10.1111/dme.12651

12. Dolan S. The technology, devices and benefits of the growing remote patient monitoring market. Business Insider. April 24, 2019. Accessed May 5. 2020. https:// www.businessinsider.in/the-technologydevices-and-benefits-of-the-growingremote-patient-monitoring-market/ articleshow/69032343.cms

13. Michaud TL, Siahpush M, King KM, et al. Program completion and glycemic control in a remote patient monitoring program for diabetes management: does gender matter? Diabetes Res Clin Pract. 2020;159:107944. doi:10.1016/j. diabres.2019.107944

14. Mushcab H, Kernohan WG, Wallace J, Harper R, Martin S. Self-management of diabetes mellitus with remote monitoring. Int J E Health Med Comm. 2017;8(1):52-61. doi:10.4018/ijehmc.2017010104

15. Odom JM, Stancil M, Nelson B, et al. Improving diabetes control through remote glucose monitoring in a diabetes self-management program for employees of a health system. Clin Diabetes. 2018;37(3):203-10. doi:10.2337/cd18-0056

16. Michaud TL, Siahpush M, Estabrooks P, et al. Association between weight loss and glycemic outcomes: a post hoc analysis of a remote patient monitoring program for diabetes management. Telemed J E Health. 2020;26(5):621-28. doi:10.1089/ tmj.2019.0030

17. Choudhry NK, Isaac $\mathrm{T}$,

Lauffenburger JC, et al. Effect of a remotely delivered tailored multicomponent approach to enhance medication taking for patients with hyperlipidemia, hypertension, and diabetes. JAMA Int Med. 2018;178(9):1182. doi:10.1001/ jamainternmed.2018.3189

18. Hamine S, Gerth-Guyette E, Faulx D, Green BB, Ginsburg AS. Impact of mHealth chronic disease management on treatment adherence and patient outcomes: a systematic review. J Med Internet Res. 2015;17(2):e52. 
19. Katalenich B, Shi L, Liu S, et al. Evaluation of a remote monitoring system for diabetes control. Clin Ther. 2015;37(6):1216-25.

20. Janis IB, Nock MK. Behavioral forecasts do not improve the prediction of future behavior: a prospective study of self-injury. J Clin Psychol. 2008;64(10):1164-74.

21. Express Scripts. 2016 drug trend report. February 2017. Accessed August 17, 2020. https://corporate-site-labs-dev. s3.amazonaws.com/2019-09/Express\%20 Scripts\%202016\%20Drug\%20Trend\%20 Report_0.pdf.

22. Sokol MC, McGuigan KA, Verbrugge RR, Epstein RS. Impact of medication adherence on hospitalization risk and healthcare cost. Med Care. 2005;43(6):521-30.

23. Bitton A, Choudhry NK, Matlin OS, Swanton K, Shrank WH. The impact of medication adherence on coronary artery disease costs and outcomes: a systematic review. Am J Med. 2013;126(4):357. e7-357.e27.

24. Osterberg L, Blaschke T. Adherence to medication. N Engl J Med. 2005;353(5):487-97.
25. DiMatteo MR, Giordani PJ, Lepper HS, Croghan TW. Patient adherence and medical treatment outcomes: a meta-analysis. Med Care. 2002;40(9):794-811.

26. Beckles GL, Engelgau MM, Narayan KM, Herman WH, Aubert RE, Williamson DF. Population-based assessment of the level of care among adults with diabetes in the U.S. Diabetes Care. 1998;21(9):1432-38.

27. Currie CJ, Peyrot M, Morgan CL, et al. The impact of treatment noncompliance on mortality in people with type 2 diabetes. Diabetes Care. 2012;35(6):1279-84.

28. Ho PM, Rumsfeld JS, Masoudi FA, et al. Effect of medication nonadherence on hospitalization and mortality among patients with diabetes mellitus. Arch Intern Med. 2006;166(17):1836-41.

29. Jha AK, Aubert RE, Yao J, Teagarden JR, Epstein RS. Greater adherence to diabetes drugs is linked to less hospital use and could save nearly \$5 billion annually. Health Aff (Millwood). 2012;31(8):1836-46.
30. Breitscheidel L, Stamenitis S Dippel FW, Schoffski O. Economic impact of compliance to treatment with antidiabetes medication in type 2 diabetes mellitus: a review paper. J Med Econ. 2010;13(1):8-15.

31. Kang H, Lobo JM, Kim S, Sohn MW. Cost-related medication non-adherence among U.S. adults with diabetes. Diabetes Res Clin Pract. 2018;143:24-33.

32. Tomlinson M, Rotheram-Borus MJ, Swartz L, Tsai AC. Scaling up mHealth: where is the evidence? PLoS Med. 2013;10(2):e1001382.

33. Viswanathan $\mathrm{M}$, Golin CE, Jones CD, et al. Interventions to improve adherence to self-administered medications for chronic diseases in the United States: a systematic review. Ann Intern Med. 2012;157(11):785-95.

34. Cutrona SL, Choudhry NK, Fischer MA, et al. Targeting cardiovascular medication adherence interventions. J Am Pharm Assoc (2003). 2012;52(3):381-97.

35. Nieuwlaat R, Wilczynski N, Navarro T, et al. Interventions for enhancing medication adherence. Cochrane Database Syst Rev. 2014;2014(11):CD000011. 